\title{
Learning an Orchestra Conductor's Technique Using a Wearable Sensor Platform
}

\author{
Dominik Schmidt \\ Ludwig-Maximilians-Universität \\ schmidte@cip.ifi.lmu.de \\ Asim Smailagic \\ Carnegie Mellon University \\ asim@cs.cmu.edu
}

\author{
Roger B. Dannenberg \\ Carnegie Mellon University \\ rbd@cs.cmu.edu \\ Daniel P. Siewiorek \\ Carnegie Mellon University \\ dps@cs.cmu.edu
}

\author{
Bernd Brügge \\ Technische Universität München \\ bruegge@in.tum.de
}

\begin{abstract}
Our study focuses on finding new input devices for a system allowing users with any skill to configure and conduct a virtual orchestra in real-time. As a first step, we conducted a user study to learn more about the interaction between a conductor's gestures and the orchestra's reaction.

During an orchestra rehearsal session, we observed a conductor's timing and gestures using the eWatch, a wrist-worn wearable computer and sensor platform. The gestures are analyzed and compared to the music of the orchestra.
\end{abstract}

\section{Introduction}

Pinocchio [2] is a system that allows users with any skill to configure and conduct a virtual orchestra in realtime, using audio and video material based on professional recording sessions with the Bavarian Radio Symphony Orchestra. In its current stage, Pinocchio recognizes common conducting gestures using a video-based approach that applies neural networks in order to control tempo and dynamics. We decided to use the eWatch [8] as an additional input device for acceleration based gesture recognition. Built into a wrist watch form factor, it is an unobtrusive wearable sensing and computing platform that does not constrain the conductor's movements.

\section{Related Work}

Mathews created the first computer system with a conducting interface using the Mechanical Baton (Daton) [7]. Lee et. al. [5] applied neural networks to recognize gestures captured by an optical tracking system. Accelerometers and Hidden Markov Models for conducting gesture recognition were used by Usa and Mochida [9]. The Media Computing Group at RWTH Aachen University developed different versions of their virtual orchestra [3]. Marrin [6] developed a Conductor's Jacket that records physiological and motion information about conductors. Baird and Irmirli [1] used a position sensor to track conducting gestures during live performances. Lee et. al. [4] conducted experiments in a controlled environment with a passive system. In contrast to systems that rely on visual approaches, the eWatch does not require any additional setup, works under different light conditions, and allows the conductor to move around freely. Furthermore, the eWatch is using low cost components and has the capability of executing recognition tasks locally. The conductor has the advantage of using his or her familiar baton.

\section{Data Collection and Analysis}

We asked conductor Daniel Meyer to wear the eWatch on his right wrist during a rehearsal. In parallel, we recorded him using a Panasonic DVC-30 digital camcorder providing video at $59.94 \mathrm{~Hz}$ and audio 
at $48 \mathrm{kHz}$. The eWatch recorded light and acceleration data at $100 \mathrm{~Hz}$. A LED emitting bright flashes that are clearly recognizable in the video track and in the light sensor data is used to synchronize eWatch and camcorder, thus accommodating for clock drift. Figure 1 depicts the system diagram with recording setup and analysis part.

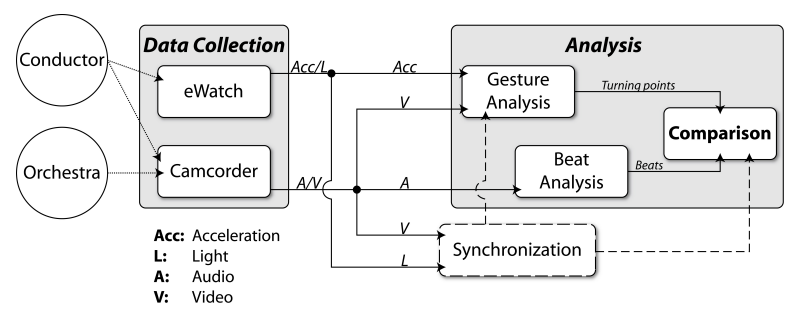

Figure 1. System diagram

A two minute long section from Brahm's Symphony No. 3 in F major, Allegro is selected for further analysis. Annotation of lower turning points in the conducting gestures (indicating the start of a measure) is done manually by observing the recorded video on a field-by-field basis. In Figure 2, velocity data of the $\mathrm{x}$ axis extracted from the eWatch's acceleration sensors is shown together with the annotations. The beat pattern is clearly recognizable.



Figure 2. Velocity and gesture annotations

As a reliable audio beat extraction tool is not available, we marked beats manually by first tapping along with the music and later applying fine adjustment using an audio editor. Similar to the results presented by Lee et. al. [4] we found that the conductor conducts ahead of the beat, showing that these results also apply to a setting where the conductor can influence the performance. At a tempo of $72 \mathrm{bpm}$ in the selected piece the conductor was ahead by $272 \mathrm{~ms}$ on average.

\section{Conclusion and Future Work}

The results obtained in this study affirm that the eWatch is a suitable and unobtrusive input device for this virtual orchestra system. Our goal is to build an user-independent and accurate recognition module that allows for the detection of arbitrary conducting gestures. The eWatch can be used as alternative or exten- sion to already existent input capabilities. We are evaluating the use of Hidden Markov Models for the recognition of continuous gestures based on acceleration data. We wish to thank conductor Daniel Meyer for allowing us to record conducting gestures during a rehearsal.

\section{Acknowledgment}

This material is based upon work supported by the National Science Foundation under Grant No. EEEC540865 and Grant No. 0205266, the Defence Advanced Project Agency (DARPA) under Contract No. NBCHD030010, Pennsylvania Infrastructure Technology Alliance (PITA) — a collaboration among Commonwealth of Pennsylvania, Carnegie Mellon and Lehigh University, and a grant from Intel Corporation.

\section{References}

[1] B. Baird and O. Izmirli. Modeling the tempo coupling between an ensemble and the conductor. In Proceedings of the International Computer Music Conference, pages 163-166, 2001.

[2] B. Bruegge, C. Teschner, P. Lachenmaier, E. Fenzl, D. Schmidt, and S. Bierbaum. Pinocchio: Conducting a virtual symphony orchestra. In Proceedings of the International Conference of Advances in Computer Entertainment Technology, 2007.

[3] E. Lee, H. Kiel, S. Dedenbach, I. Grüll, T. Karrer, M. Wolf, and J. Borchers. In CHI '06: CHI'O6 Extended Abstracts on Human Factors in Computing Systems.

[4] E. Lee, M. Wolf, and J. Borchers. Improving orchestral conducting systems in public spaces: examining the temporal characteristics and conceptual models of conducting gestures. In CHI '05: Proceedings of the SIGCHI Conference on Human Factors in Computing Systems, pages 731-740, 2005.

[5] M. Lee, G. Garnett, and D. Wessel. An adaptive conductor follower. In Proceedings of the International Computer Music Conference, pages 454-455, 1992.

[6] T. Marrin and R. Picard. The "Conductor's Jacket": A device for recording expressive musical gestures. In Proceedings of the International Computer Music Conference, pages 215-219, 1998.

[7] M. V. Mathews. The Conductor Program and Mechanical Baton, pages 263-281. 1989.

[8] U. Maurer, A. Rowe, A. Smailagic, and D. P. Siewiorek. eWatch: A wearable sensor and notification platform. In Wearable and Implantable Body Sensor Networks, 2006. BSN 2006., 2006.

[9] S. Usa and Y. Mochida. A multi-modal conducting simulator. In Proceedings of the International Computer Music Conference, pages 25-32, 1998. 